\title{
Time-Frequency Analysis of Vibration Propagation from Intima to Adventitia of Arterial Wall
}

\author{
Kazuhiro Sunagawa ${ }^{\mathbf{1}, 2}$, Hiroshi Kanai ${ }^{1}$, Yoshiro Koiwa ${ }^{3}$ and Motonao Tanaka ${ }^{4}$ \\ 1 Dept. of Electrical Eng., Graduate School of Eng., Tohoku University, Sendai 980-8579, Japan \\ 2 Matsushita Communication Sendai R\&D Labs. Co., Ltd., Sendai 981-3206, Japan \\ 3 Dept. of Internal Medicine, Graduate School of Medicine, Tohoku University, Sendai 980-8574, Japan \\ 4 Tohoku Welfare Pension Hospital, Sendai 983-8512, Japan
}

\begin{abstract}
Based on the phased tracking method, it is newly found that there are wide frequency components from $\mathrm{d}$. c. to one hundred several tens $\mathrm{Hz}$ in the vibrations on the arterial wall non-invasively measured in in vivo experiments. These vibrations are mainly caused by the blood flow, and the wall vibration propagates from the intima to the adventitia. Moreover, the characteristics of the arterial wall vibration highly depend on the viscoelasticity of tissue components.

In this paper, therefore, the propagation of the vibration in the regional area of the arterial wall is analyzed during one cardiac cycle in the frequency domain. From the measurement of the frequency characteristics in the vibration propagation, the tissue viscoelasticity is estimated. In the low frequency range, both the attenuation and the phase of the vibration are minute values, therefore, it is so difficult to measurement of these precisely. For solving this problem, the viscoelasticity of the arterial wall is estimated by the frequency characteristics in the attenuation in the wide frequency range up to one hundred several tens $\mathrm{Hz}$. The proposed method was applied to human common carotid arteries of two patients with atherosclerotic plaques, a smoking subject and a healthy subject. From in vivo experimental results, clear differences are found in the the frequency characteristics in the attenuation and the estimated viscoelasticity of the arterial wall between atherosclerotic plaque of the patient and healthy subject. These results has novel potential for the tissue characterization.
\end{abstract}

\section{INTRODUCTION}

It is well known that acute myocardial infarction and cerebral infarction are mainly caused by rupture of atherosclerotic plaques of the artery. It is, therefore, necessary for clinical treatment to be able to discriminate thrombus, fibrous, calcification and lipid components of atherosclerotic plaques [1], [2].

We have previously developed a novel method, namely, the phased tracking method, for accurately tracking the movement of the heart wall based on both the phase and magnitude of the demodulated signals to determine the instantaneous position of an object [3].

Based on this method, it is newly found that there are wide frequency components from d. c. to one hundred several tens $\mathrm{Hz}$ in the vibrations on the arterial wall non-invasively measured in in vivo experiments [4].

These vibrations are mainly caused by the blood flow, and the wall vibration propagates from the intima to the adventitia. Moreover, the characteristics of the arterial wall vibration highly depend on the viscoelasticity of the tissue components. Therefore, from the viscoelasticity, it would offer a clue to characterize the human tissue.

At the human tissue, the characteristics of vibration propagation have the frequency characteristics[5]. Based on Voigt model, the viscoelasticity can be estimated from the vibration frequency, the attenuation and the phase.

However, in the low frequency range, both the attenuation and the phase are minute values, it is so difficult to measurement of these precisely.

For solving this problem, the viscoelasticity of the arterial wall is estimated by the frequency characteristics in the attenuation in the wide frequency range up to one hundred several $\mathrm{Hz}$.

The proposed method was applied to human common carotid arteries of two patients with atherosclerotic plaques, a smoking subject and a healthy subject.

\section{Frequency Characteristics in attenuation of Shear Wave Propagation in Human Tissue}

Figure 1 shows illustration of vibration propagation from the intima to the adventitia in the arterial wall. At the inner surface of artery, wide frequency range vibrations are occurred by change in the blood pressure and the blood flow, and the vibration propagate from the intima to the adventitia with the shear wave.

For modeling of the vibration propagation in the hu- 


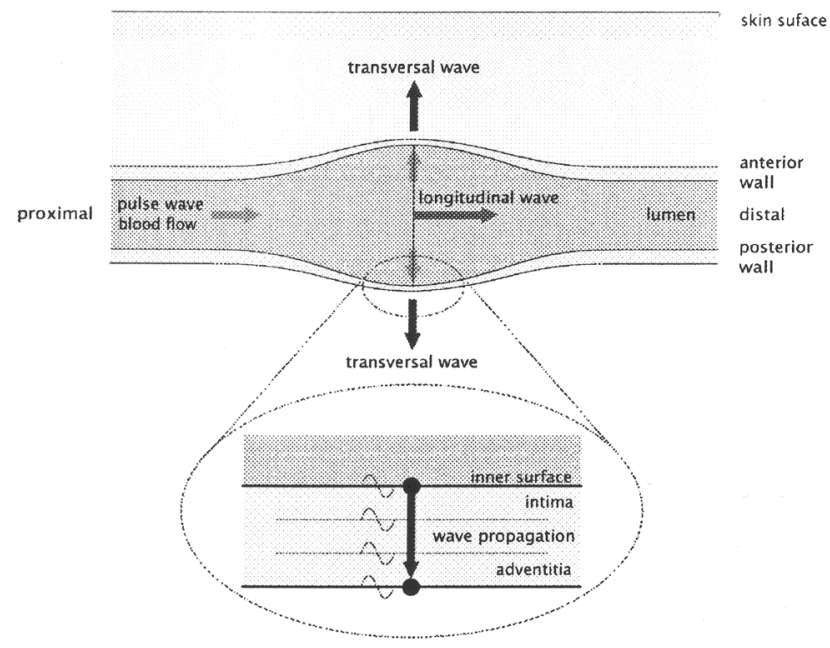

Fig. 1. Mlustration of the shear wave propagating from the intima to the adventitia in the arterial wall.

man tissue, Voigt model is applied to equation of plane transversal wave. In this model, the vibration velocity $v(x)$ given by

$$
V(x)=V_{0} e^{-\gamma x},
$$

where $x$ is distance from the vibration source and $v_{0}$ is vibration velocity at the vibration source.

In Eq. (1), the relationship among the attenuation $\alpha$, phase $\beta$ and the viscoelasticity $G$ is obtained by

$$
-\gamma=-(\alpha+j \beta)=-j \sqrt{\frac{\rho}{G}} \omega,
$$

where $\rho$ is density of the tissue, and $\omega$ is the vibration angular frequency.

From Eq. (3), the viscoelasticity $G$ is given by

$$
G=\mu+j \omega \eta=-\frac{\rho \omega^{2}}{\gamma^{2}} .
$$

From Eq. (3), the attenuation $\alpha$ and phase $\beta$ are described by the elasticity $\mu$ and the viscosity $\eta$ as follows:

$$
\begin{aligned}
& \alpha(\omega)=\left(\frac{\rho \omega^{2}\left(\sqrt{\mu^{2}+\omega^{2} \eta^{2}}-\mu\right)}{2\left(\mu^{2}+\omega^{2} \eta^{2}\right)}\right)^{\frac{1}{2}}, \\
& \beta(\omega)=\left(\frac{\rho \omega^{2}\left(\sqrt{\mu^{2}+\omega^{2} \eta^{2}}+\mu\right)}{2\left(\mu^{2}+\omega^{2} \eta^{2}\right)}\right)^{\frac{1}{2}} .
\end{aligned}
$$

From Eq. (4) and Eq. (5), the attenuation $\alpha$ and the phase $\beta$ are function of the angular frequency $\omega$.

\section{Estimation of Shear Viscoelasticity From Frequency CharaCteristics in Attenuation of Share Wave Propagation}

On the other hand, from Eq. (3), the elasticity $\mu$ and viscosity $\eta$ are described by the attenuation $\alpha$ and the phase $\beta$ as follows:

$$
\begin{gathered}
\mu=\frac{\rho \omega^{2}\left(\alpha^{2}-\beta^{2}\right)^{2}}{\left(\alpha^{2}+\beta^{2}\right)^{2}}, \\
\eta=\frac{2 \rho \omega \alpha \beta}{\left(\alpha^{2}+\beta^{2}\right)^{2}} .
\end{gathered}
$$

A case of human muscle tissue, $\rho=1.1 \times 10^{3} \mathrm{~kg} / \mathrm{m}^{3}$, $\mu=2500 \mathrm{~Pa}, \eta=15.0 \mathrm{~Pa} \cdot \mathrm{s}$ [5], in the frequency at $100 \mathrm{~Hz}$, the attenuation and phase are $\alpha=129 \mathrm{~m}^{-1}$, $\beta=170 \mathrm{rad} / \mathrm{m}$, and in the frequency at $20 \mathrm{~Hz}$, these are $\alpha=24 \mathrm{~m}^{-1}, \beta=71 \mathrm{rad} / \mathrm{m}$.

At the human carotid arterial wall, in the thickness $\Delta x$ at about $1 \mathrm{~mm}$, in the frequency at $20 \mathrm{~Hz}$, the attenuation and the phase are $\Delta x \cdot \alpha=0.02 \mathrm{~m}^{-1}$, $\Delta x \cdot \beta=0.07 \mathrm{rad} / \mathrm{m}$.

In the low frequency range, therefore, it is difficult to measurement of the attenuation and phase precisely.

For solving this problem, the viscoelasticity is estimated by the frequency characteristics in the attenuation of the vibration propagation in the wide frequency range up to one hundred several tens $\mathrm{Hz}$.

In Eq. (4), the attenuation $\alpha$ is function of the angular frequency $\omega$, the elasticity $\mu$ and the viscosity $\eta$. Moreover, in Eq. (4), the elasticity $\mu$ and the viscosity $\eta$ are unknown quantities.

Therefore, the elasticity $\mu$ and the viscosity $\eta$ are assumed to be constant with change in the frequency.

From Eq. (4), the squared attenuation $\alpha(\omega)^{2}$ is given by

$$
\begin{aligned}
& \alpha(\omega)^{2}= \frac{\rho \omega^{2}\left(\sqrt{\mu^{2}+\omega^{2} \eta^{2}}-\mu\right)}{2\left(\mu^{2}+\omega^{2} \eta^{2}\right)} \\
&= \rho \omega^{2} \sqrt{\omega^{2} \eta^{2}}\left(\sqrt{1+\frac{\mu^{2}}{\omega^{2} \eta^{2}}}-\frac{\mu}{\omega \eta}\right) \\
& \times \frac{1}{2} \frac{1}{\omega^{2} \eta^{2}\left(1+\frac{\mu^{2}}{\omega^{2} \eta^{2}}\right)} \rho \omega \eta\left\{\left(1+\frac{\mu^{2}}{\omega^{2} \eta^{2}}\right)^{\frac{1}{2}}-\frac{\mu}{\omega \eta}\right\} \\
& \times \frac{1}{2 \eta^{2}}\left(1+\frac{\mu^{2}}{\omega^{2} \eta^{2}}\right)^{-1}
\end{aligned}
$$

In the high frequency range to $\omega^{2} \eta^{2}>>\mu^{2}$, Eq. (8) is approximated as follows: 


$$
\begin{aligned}
\alpha(\omega)^{2} & \approx \frac{\rho \omega}{2 \eta}\left(1+\frac{\mu^{2}}{2 \omega^{2} \eta^{2}}-\frac{\mu}{\omega \eta}\right)\left(1-\frac{\mu^{2}}{\omega^{2} \eta^{2}}\right) \\
& \approx \frac{\rho \omega}{2 \eta}\left(1-\frac{\mu}{\omega \eta}\right) .
\end{aligned}
$$

From Eq. (9), in the high frequency range, the squared attenuation $\alpha(\omega)^{2}$ is approximated to linear function of the angular frequency $\omega$ as follows:

$$
\widehat{\alpha}(\omega)^{2}=\frac{\rho}{2 \eta} \omega-\frac{\rho \mu}{2 \eta^{2}}=A \omega+B
$$

From Eq. (10), the elasticity $\hat{\mu}$ and the viscosity $\hat{\eta}$ can be estimated by

$$
\widehat{\eta}=\frac{\rho}{2 A}, \quad \widehat{\mu}=-\frac{2 \eta^{2} B}{\rho} .
$$

\section{Measurement of viscoelasticity of HUMAN CAROTID ARTERY}

Let us set measurement points at the intima and the adventitia in the arterial wall along an ultrasonic beam, and by applying the phased tracking method to each point, the velocity signals of the each point is simultaneously measured.

For this measurement, the frequency $f_{0}$ of ultrasonic pulse was set to be $7.5 \mathrm{MHz}$ and pulse repetition frequency was set to be $1.5 \mathrm{kHz}$ for respective directions.

By applying the Fourier transform to each vibration signal during one cardiac cycle, the spectra of the vibration signals are obtained.

This measurements was applied to the human common carotid arteries of two patients with atherosclerotic plaques, a smoker and a healthy subject.

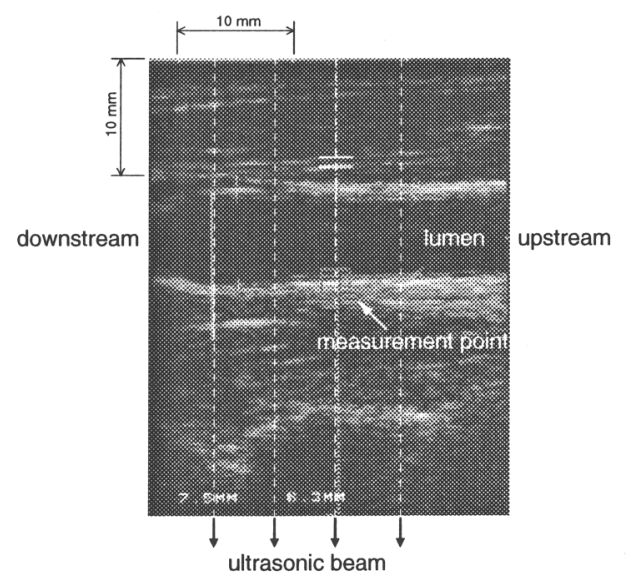

Fig. 2. B-mode image of a human carotid artery (healthy subject, 28-year-old male)
Figures 2, 3, 4, 5 are B-mode images of the human carotid artery. As shown in Figs. 3, 4, there are atherosclerotic plaques at the posterior wall.

Figure 6 shows measurement results of the frequency characteristics in the squared attenuation of the vibration propagation from the intima to the adventitia in the ejection period.

In Fig. 6, solid lines are measurement results and dotted lines are approximated lines by the least-squared approach in the high frequency range.

For applying the least-squared approach, the high frequency range was set to higher $50 \mathrm{~Hz}$ from referred human muscle tissue [5].

As shown in Fig. 6, in the frequency range higher than $50 \mathrm{~Hz}$, measurement results of squared attenuation are linearly increased with frequency increment, and the approximated lines are closely fitted to the measurements.

From the approximated lines, the elasticity $\hat{\mu}$ and the viscosity $\hat{\eta}$ are estimated by Eq. (11).

Table 1 shows estimated results of $\hat{\mu}$ and $\hat{\eta}$. There are clear difference of the estimated viscoelasticity between healthy subject and patients with plaque. For the healthy subject and smoking subject, estimated viscoelasticity are similar to those of referred human muscle tissue [5]. However, for the patient with plaque, estimated viscoelasticity are smaller than those of healthy subject, smoking subject and referred data.

Figure 7 shows the frequency characteristics in the attenuation are calculated by Eq. (4) using estimated viscoelasticity as shown in table 1 .

As shown in Fig. 7, solid lines are measurement results of the frequency characteristics in the attenuation and dotted lines are estimated results. These results

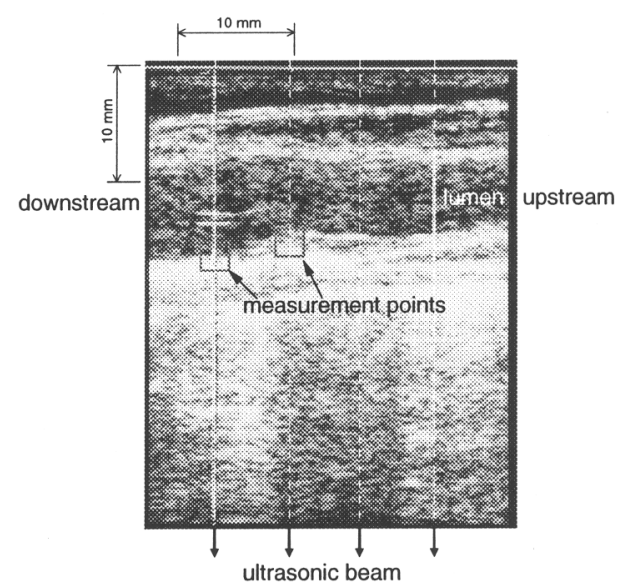

Fig. 3. B-mode image of a human carotid artery (patient A, 40-year-old, female) 
are closely fitted to measurement results.

\section{CONCLUSIONS}

Based on in vivo experiments, the propagation characteristics of the vibration from the intima to the adventitia of the arterial wall is measured in high frequency range up to $150 \mathrm{~Hz}$.

From frequency characteristics in attenuation of vibration propagation from the intima to the adventitia in the wide frequency range, the viscoelasticity is approximately estimated.

From the results, the proposed method might be reasonable for estimation of the viscoelasticity of the arterial wall tissue.

These results has novel potential for the tissue characterization.

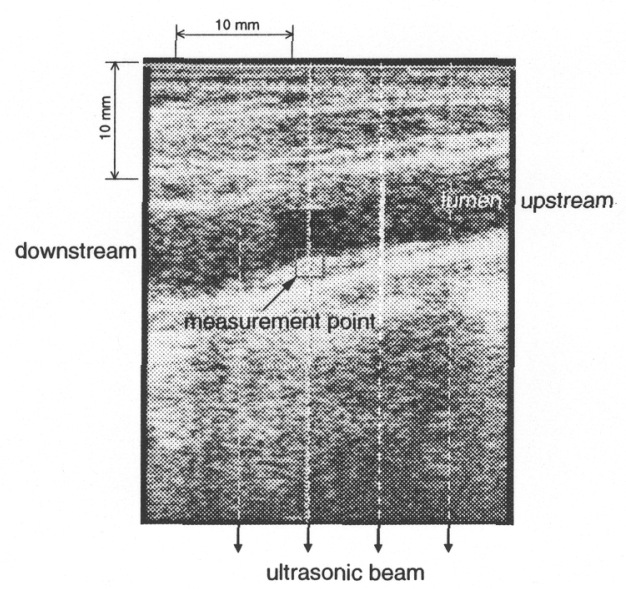

Fig. 4. B-mode image of a human carotid artery (patient B, 73-year-old, male)

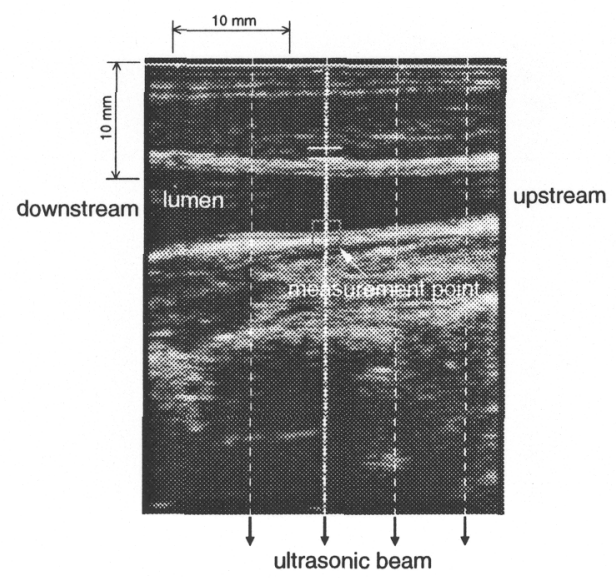

Fig. 5. B-mode image of a human carotid artery (smoking subject, 30-year-old, male)

\section{ReFERENCES}

[1] European carotid surgery trialist' collaborative group (ECST): MRC European carotid surgery trial: Lancet $\mathbf{3 3 7}$ (1991) 1235.

[2] North American symptomatic carotid endarterectomy trial collaborators (NASCET): New Eng. J. Med. 325 (1991) 445.

[3] H. Kanai, M. Sato, Y. Koiwa and N. Chubachi: IEEE Trans. on UFFC 43 (1996) 791.

[4] K. Sunagawa, H. Kanai, Y. Koiwa, K. Nitta and M. Tanaka: 1999 IEEE International Ultrasonics Symposium Proceedings (1999) 1507.

[5] H. L. Oestreicher: J. A coust. Soc. Am. 37(2) (1951) 707714.

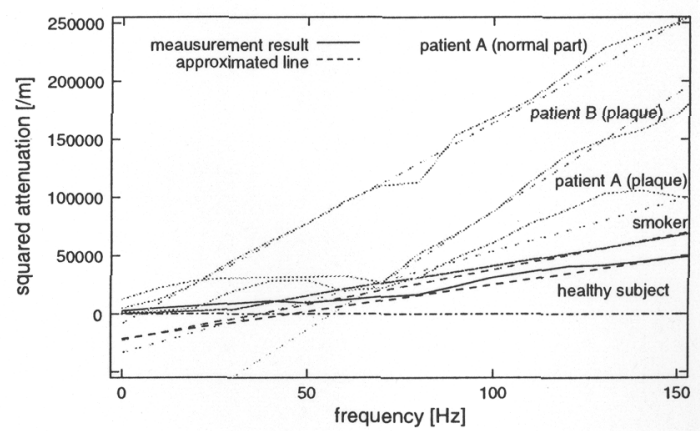

Fig. 6. Frequency characteristics in the attenuation of the vibration propagation from the intima to the adventitia.

TABLE I

RESUlts OF THE ESTIMATION OF THE ELASTICITY, $\widehat{\mu}$, AND VISCOSITY, $\widehat{\eta}$

\begin{tabular}{|l||r|r|}
\hline & $\widehat{\mu}[\mathrm{Pa}]$ & $\widehat{\eta}[\mathrm{Pa} \cdot \mathrm{s}]$ \\
\hline \hline healthy subject & 2100 & 7.4 \\
\hline smoking subject & 1300 & 5.7 \\
\hline patient A (normal part) & 60 & 2.0 \\
\hline patient A (plaque) & 960 & 4.0 \\
\hline patient B (plaque) & 610 & 1.7 \\
\hline \hline referred data [5] & 2500 & 15.0 \\
\hline
\end{tabular}

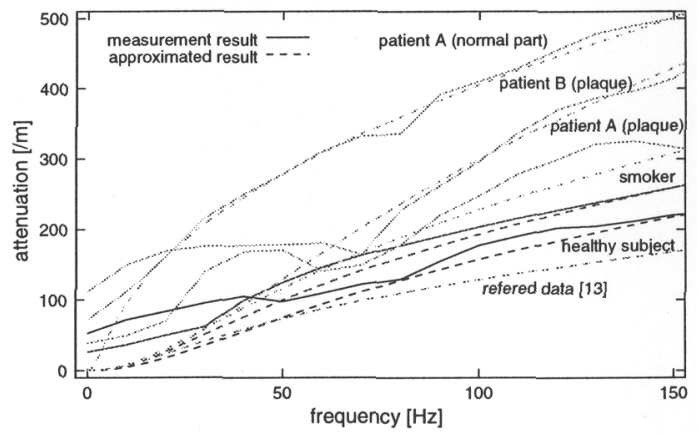

Fig. 7. Measurement results of the frequency characteristics in the squared attenuation and the approximated lines by the proposed method. 